\title{
COMPARISON OF DIFFERENT PADC MATERIALS FOR NEUTRON DOSIMETRY
}

\author{
S. Mayer* and M. Boschung \\ Division for Radiation Safety and Security, Paul Scherrer Institute (PSI), Villigen PSI, Switzerland \\ *Corresponding author: sabine.mayer@psi.ch
}

\begin{abstract}
Investigations on track density and track size distributions of different PADC (poly allyl diglycol carbonate) materials have been performed. The PADC used for the tests has been produced by Thermo Electron (USA), Track Analysis System Limited (UK), Chiyoda Technol Corporation (Japan) and Intercast srl (Italy). For each PADC material 120 detectors were randomly selected out of 2 sheets: 60 detectors from one sheet have been irradiated with a personal dose equivalent of $3 \mathrm{mSv}$ in the field of a ${ }^{241} \mathrm{Am}$-Be source at the calibration laboratory of PSI, whilst the other 60 detectors from the other sheet have been used as background samples. All detectors have been processed according to an identical etching procedure and have been analysed with TASLImage scanning system. For each set of detectors the value of the average background signal, the average neutron sensitivity and the detection limit with respect to a personal dose equivalent measured with a dosemeter based on PADC have been determined. The results of the investigations allowed a comparison of the neutron sensitivity and background signal behaviours of PADC materials from different manufacturers and the assessment of the variation of neutron sensitivity and background signal over a single sheet.
\end{abstract}

\section{INTRODUCTION}

Solid-state neutron track dosimetry on the basis of PADC (poly allyl diglycol carbonate) material has been in operation at PSI since $1998^{(1)}$. Quality assurance on the PADC material is applied routinely to guarantee reliable personal dosimetry. One part of the quality assurance is the acceptance test where the neutron sensitivity and background signal is checked and compared with established acceptance criteria. The PADC material is normally delivered in sheets of $\sim 100$ or 200 single detector pieces. For practical and economic reasons only $\sim 8 \%$ of the detectors are used to perform the quality assurance. The representativeness of the sample size for assessing the performance of all detectors from one sheet is therefore crucial. Hence, the batch-to-batch and even sheet-to-sheet variability in both neutron sensitivity and background is a major issue for the routine application of track etch detectors with the PADC material in personal neutron dosimetry. Of particular importance has been in the past the problem of occasional large variance of mean sheet background and other properties affecting the background.

With the availability of a microscope-based automatic scanning technique (TASLImage scanning system) the characterisation of tracks became more comprehensive and the analysis more stringent. By using several specific track characteristics such as size, shape and optical density and their distributions and by comparing them with the distributions of a reference set the discrimination of background tracks or noise was improved. Therefore, the influence of material inhomogeneity should no longer be a problem. The goal of the present study was to

- test the performance of the TASLImage track analysis system regarding the latter described feature;

- study the variability in both sensitivity and background for five different PADC materials to support the routine quality assurance program;

- assess the equivalence of the five different PADC materials for determining the personal neutron dose with the PSI routine evaluation procedure.

\section{MATERIALS AND METHODS}

\section{Detector description}

The PADC material used for the tests has been produced by Thermo Electron (USA), Track Analysis System Limited TASL (UK), Chiyoda Technol Corporation (two systems, Japan) and Intercast srl (Italy).

The material by Thermo Electron of type PN3, denoted hereafter 'TE', comes in sheets of 231 detectors per sheet. Each detector has a rectangular shape $\left(2.0 \times 2.5 \mathrm{~cm}^{2}\right)$ and a thickness of $0.16 \mathrm{~cm}$. TASL provides TASTRAK ${ }^{\mathrm{TM}}$ material, denoted hereafter 'TASL', which is produced in sheets of $31 \times 26 \mathrm{~cm}^{2}$ and then laser cut to any dimension and shape. For the purposes of authors one sheet is divided into 117 detectors with a rectangular shape $\left(2.0 \times 2.5 \mathrm{~cm}^{2}\right)$ and a thickness of $0.15 \mathrm{~cm}$. The material of Chiyoda Technol Corporation is called TechnoTrak ${ }^{\mathrm{TM}}$. It is available in two different qualities, denoted hereafter 'Tech1' and 
'Tech2' (new formulation). One sheet consists of 99 detectors with dimensions of $2.0 \times 2.5 \mathrm{~cm}^{2}$ and a thickness of $0.13 \mathrm{~cm}$, laser cut by the manufacturer. The material of Intercast, denoted hereafter 'Inter', comes in sheets of $26 \times 31 \mathrm{~cm}^{2}$ from which 132 detectors with a rectangular shape $\left(2.0 \times 2.5 \mathrm{~cm}^{2}\right)$ and a thickness of $0.17 \mathrm{~cm}$ are cut out by the manufacturer.

Note that the number of detectors does not correspond exactly to the finally analysed number, since some detectors were already excluded due to visible artefacts on the surface.

\section{Etching procedure and evaluation}

After exposure and before evaluation, the PADC detectors have to undergo a chemical etching process. The same etching procedure was applied for the five materials. Only for the Tech1 material an additional pre-soaking treatment was applied according to the suggestion of the manufacturer ${ }^{(2)}$. The formulation of the Tech 2 material has been chosen by the manufacturer to avoid the pre-soaking step. The detectors were etched for $2 \mathrm{~h} 50 \mathrm{~min}$ at $85^{\circ} \mathrm{C}$ in $6.25 \mathrm{M}$ sodium hydroxide. The chemical etching process was routinely monitored by registering the temperature, which was stabilised to $\pm 1^{\circ} \mathrm{C}$. Subsequently they were neutralised in a weak hydrochloric acid solution for $15 \mathrm{~min}$ and washed with hot $\left(60^{\circ}\right)$ and cold (room temperature) distilled water for $10 \mathrm{~min}$, respectively. After that the detectors were dried at $40^{\circ}$ in an oven for $1 \mathrm{~h}$ the tracks on the detectors were then counted with the TASLImage system $^{(3)}$.

\section{Irradiation procedure}

For each material 60 detectors have been randomly selected from one sheet and irradiated in the field of a ${ }^{241} \mathrm{Am}$ - Be source at the accredited calibration laboratory at $\mathrm{PSI}^{(4)}$ with a personal dose equivalent $H_{\mathrm{p}}(10)$ of $3 \mathrm{mSv}$ in the standard PSI neutron dosemeter. The other 60 randomly selected detectors from the second sheet have been used as background detectors.

\section{Data analysis}

For each material the average number of net tracks per $\mathrm{cm}^{-2}$ of the irradiated detectors, $N$, and the average number of tracks per $\mathrm{cm}^{-2}$ of the background detectors, $B$, as well as the average neutron sensitivity in tracks per $\mathrm{cm}^{-2}$ per unit personal dose equivalent, $R$, and the personal dose equivalent $H_{\mathrm{LLD}}$ implied by the reading corresponding to the detection limit, $L_{\mathrm{D}}$, were determined. $H_{\text {LLD }}$ was calculated according the formula $^{(5)}$ :

$$
H_{\mathrm{LLD}} \cong \frac{4\left(\sigma_{\mathrm{B}}+1\right)}{R}
$$

where $\sigma_{\mathrm{B}}$ is the standard deviation of the background track density.

Note that $N$ is already the value after the application of the TASL evaluation algorithm. This proprietary algorithm corrects for the background and sensitivity of each single detector. In particular it takes into account alpha particle and non-track background cross contamination and the quality of scan.

\section{RESULTS AND DISCUSSION}

In Figure 1 the histograms of the frequency distributions of the sensitivity $R$ and in Figure 2 the individual background track density of non-irradiated detectors are plotted for all materials.

The comparison of different parameters, such as sensitivity $R$, net mean track densities $N$ for a dose $H_{\mathrm{p}}(10)$ of $3 \mathrm{mSv}$ from ${ }^{241} \mathrm{Am}-\mathrm{Be}$, mean background track densities $B$ and personal dose equivalent detection limit for the TASL system $H_{\mathrm{LLD}}$, is presented in Table 1. The uncertainties reported in Table 1 were determined in different ways: $\sigma_{\mathrm{N}}$ and $\sigma_{\mathrm{B}}$ were calculated as the sample standard deviation of the net track densities and background track densities, and $\sigma_{R}$ was calculated for a coverage factor $k=2$ and $95 \%$ confidence interval including the uncertainties of the measured track density of the applied reference dose $(4 \%)$ and of the calibration procedure $(3 \%)$. The uncertainty related to the track counting is in the order of $1-2 \%$ while the overall uncertainty of the calculated sensitivity $R$ is in the order of $10 \%$ for all materials.

The sensitivity $R$ to ${ }^{241} \mathrm{Am}-\mathrm{Be}$ is highest for the material Inter and lowest by almost a factor 3 for the material TASL and Tech1. With the new formulation for the material Tech 2 the sensitivity increases by 20 $\%$ compared with the material Tech1.

Figure 3 shows the mean frequency distribution of track sizes for all detectors of each material as given by the TASLImage system. The distributions marked

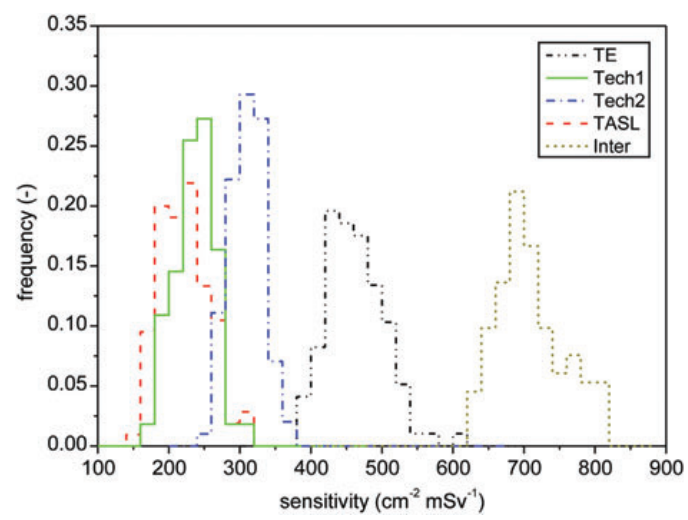

Figure 1. Histogram of frequency distributions of the sensitivity for five different PADC materials. 


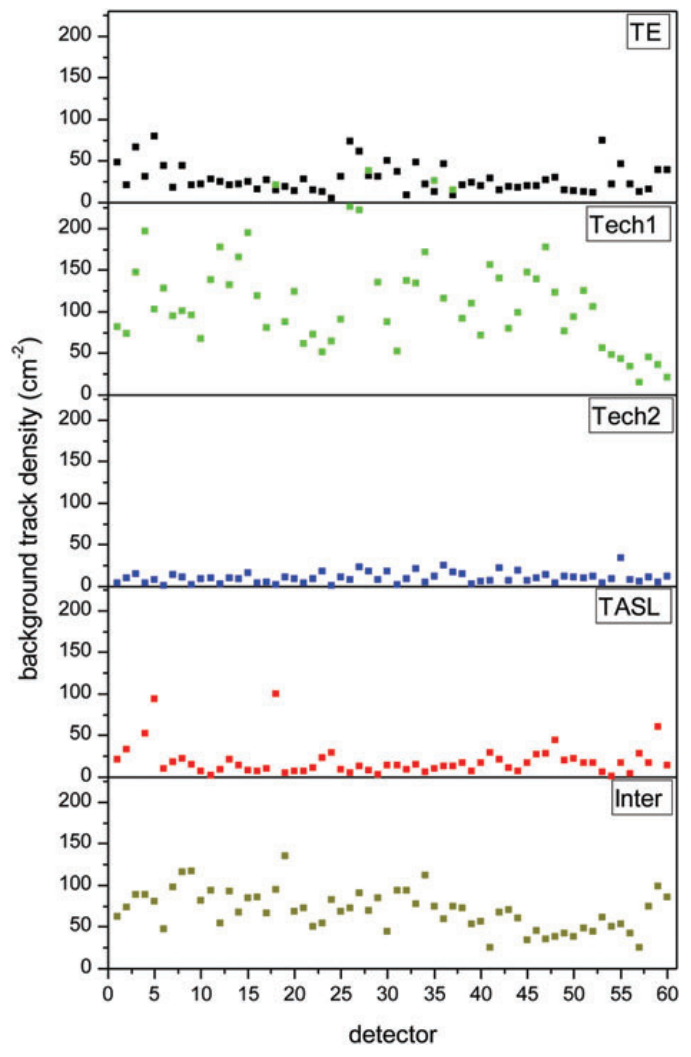

Figure 2. Background track density of non-irradiated detectors for five different PADC materials.

Table 1. Comparison of characteristic parameters (mean value and standard deviation) for five different materials evaluated with the TASLImage system. The values for $\sigma_{B}$ include the uncertainty of the evaluation algorithm (see explanation above).

\begin{tabular}{lcccc}
\hline Material & $\begin{array}{c}R \pm \sigma_{R} \text { for } \\
{ }^{241} \mathrm{Am}-\mathrm{Be} \\
\left(\mathrm{cm}^{-2} \mathrm{mSv}{ }^{-1}\right)\end{array}$ & $\begin{array}{c}N \pm \sigma_{\mathrm{N}} \\
\left(\mathrm{cm}^{-2}\right)\end{array}$ & $\begin{array}{c}B \pm \sigma_{\mathrm{B}} \\
\left(\mathrm{cm}^{-2}\right)\end{array}$ & $\begin{array}{c}H_{\mathrm{LLD}} \\
(\mathrm{mSv})\end{array}$ \\
\hline TE & $459.6 \pm 46.8$ & $1379 \pm 13$ & $29 \pm 17$ & 0.16 \\
Tech1 & $255.8 \pm 26.8$ & $767 \pm 12$ & $117 \pm 61$ & 0.97 \\
Tech2 & $316.3 \pm 32.2$ & $930 \pm 7$ & $10 \pm 7$ & 0.10 \\
TASL & $225.9 \pm 23.7$ & $678 \pm 11$ & $19 \pm 19$ & 0.35 \\
Inter & $733.0 \pm 74.4$ & $2159 \pm 14$ & $66 \pm 21$ & 0.12 \\
\hline
\end{tabular}

as 'raw' are those which the system identifies as tracks after scanning the standard area of $0.9 \mathrm{~cm}^{2}$ and the distributions marked as 'processed' are the remaining tracks after a processing step which eliminates unwanted track and surface artefacts. The 'raw' distributions are normalised to the track density of the 'processed' distributions. During the evaluation process a threshold

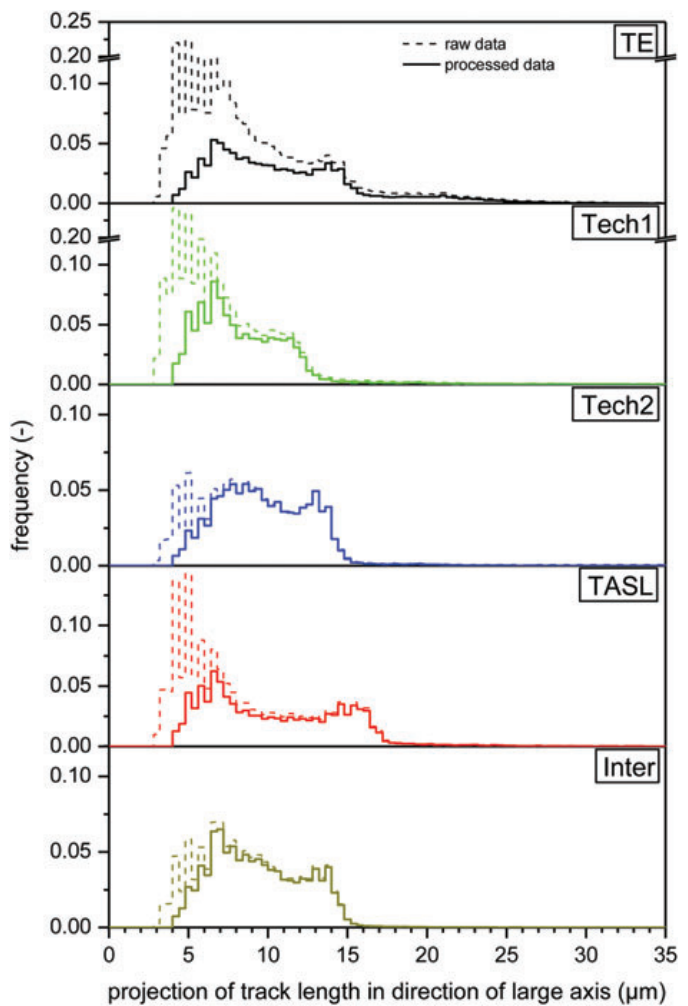

Figure 3. Track size frequency distribution of irradiated detectors for five different PADC materials (dashed line: raw data; solid line: processed data).

of $4 \mu \mathrm{m}$ to the track length as well as alpha particle and non-track background discrimination is applied. This step may also reduce the effective area to be used to calculate the final track density. It can be seen in Figure 3 that the small tracks are mostly affected by the evaluation algorithm. For the materials TE and Tech $1 \sim 50 \%$ of the initial detected tracks are rejected and for the other materials the reduction is in the order of 20-40\% (TE: $57 \%$; Tech1: $48 \%$; TASL: $36 \%$; Tech2: $28 \%$; Inter: $16 \%$ ). The maximum track length for each material varies between $\sim 14 \mu \mathrm{m}$ (Tech1) up to $\sim 25 \mu \mathrm{m}$ (TE). No correlation between maximum track length or track rejection fraction and sensitivity can be observed.

The personal dose equivalent $H_{\mathrm{LLD}}$ implied by the reading corresponding to the detection limit is 0.97 $\mathrm{mSv}$ for material Tech1 and between 0.12 and 0.35 $\mathrm{mSv}$ for the other materials. Even though the materials TE and Inter have larger sensitivities compared with the other materials, the $H_{\mathrm{LLD}}$ are almost identical, except for the material Tech1. For the latter material the track density variation of the background detectors are large (see Figure 2 ) and thus the $H_{\mathrm{LLD}}$ is 
also larger. To reduce the lower detectable dose it is not only important to have a high sensitivity but also to get a low variation on the measured tracks of background detectors.

\section{CONCLUSION}

The neutron sensitivity and the background signal of five different commercial available PADC materials were determined using the routine PSI evaluation procedure, including the routine etching procedure, the scanning system and evaluation algorithm of TASL. The differences in sensitivity of the PADC materials to ${ }^{241} \mathrm{Am}-\mathrm{Be}$ can be significant and most probably related to the formulation of the material manufacturing process. Although the sensitivities of the five studied PADC materials vary considerably, the lowest detectable dose $H_{\text {LLD }}$ remains similar. From the comparison it can be concluded that all studied PADC materials could be used for assessing the personal neutron dose with the routine PSI evaluation procedure, except the material Tech1, which yields a high value for the lowest detectable dose.

\section{FUNDING}

This work was partly supported by the Swiss Federal Nuclear Safety Inspectorate (ENSI, contract 100979).

\section{REFERENCES}

1. Fiechtner, A. and Wernli, C. Individual neutron monitoring with CR-39 at an accelerator centre. Radiat. Prot. Dosim. 85(1-4), 35-38 (1999).

2. Hiroyuki, O., Wakako, S., Keiji, O., Takashi, N. and Tonoya, Y. Charactersitics of PADC detectors using new pre-soaking technique. Radiat. Meas. 43, 437-441 (2008).

3. Fiechtner-Scharrer, A., Mayer, S., Boschung, M. and Whitelaw, A. Influence of variation of etching conditions on the sensitivity of PADC detectors with a new evaluation method. Radiat. Prot. Dosim. 144(1-4), 150-154 (2011).

4. Hoedlmoser, H., Boschung, M., Hohmann, E. and Mayer, S. Neutron reference fields at the PSI calibration laboratory. This issue of RPD.

5. Harvey, J. R., Tanner, R. J., Alberts, W. G., Bartlett, D. T., Piesch, E. K. A. and Schraube, H. The contribution of EURADOS and CENDOS to etched track neutron dosimetry: the current status in Europe. Radiat. Prot. Dosim. 77(4), 267-304 (1998). 